\title{
ON CHARACTERIZATIONS OF INNER PRODUCT SPACES
}

\section{DETLEF LAUGWITZ}

ABSTRACT. The characterizations of inner product norms given by Tapia [10] make use of a generalized inner product in normed spaces. I shall give simpler proofs and a sharper result using a more geometrical approach.

1. The generalized inner product of a norm. Let $f: V \rightarrow \mathbf{R}$ be a function defined on the real vector space $V$. Throughout the paper we shall use the following topology on $V: \lim _{n \rightarrow \infty} x_{n}=x_{0}$ for $x_{n} \in V$ iff there is a finite dimensional subspace $V_{f} \subseteq V$ containing all $x_{n}, x_{0}$ with $x_{n} \rightarrow x_{0}$ in the conventional topology of $V_{f}$.

By definition,

$$
f_{+}^{\prime}(x)(h)=\lim _{t \rightarrow 0+} \frac{f(x+t h)-f(x)}{t} \quad(h, x \in V) .
$$

The following proposition is elementary (cf. [2, p. 19]):

(a) Let $f$ be convex; then $f_{+}^{\prime}(x)(b)$ exists, and is a convex function of $b$ for every $x \in V$, and for $s>0, f_{+}^{\prime}(x)(s \cdot b)=s \cdot f_{+}^{\prime}(x)(b)$.

If \|\| is any norm on $V$, then \|\| and

$$
g: x \rightarrow\|x\|^{2} / 2
$$

are convex functions. By (2), Tapia's generalized inner product exists:

$$
(x, h)=g_{+}^{\prime}(x)(b) \quad \text { (definition). }
$$

(Note that (3) is a well-known object in differential geometry in case $g$ has good differentiability properties [4], [6], [7], [9].) If \|\| is a pre-Hilbert norm, then (3) coincides with the given inner product. In the general case we have

Received by the editors December 7, 1973 and, in revised form, April 1, 1974. AMS (MOS) subject classifications (1970). Primary 46B99; Secondary 46C10, $52 \mathrm{~A} 05$. bodies.

Key words and phrases. Inner product, differentiability of the norm, convex 


$$
(x, s \cdot h)=(s x, h)=s \cdot(x, h) \text { for } s>0
$$

and

$$
(x, x)=\|x\|^{2} \text {. }
$$

From

$$
f_{+}^{\prime \prime}(x)\left(h_{1} ; h_{2}\right):=\lim _{t \rightarrow 0+} \frac{1}{t}\left\{f_{+}^{\prime}\left(x+t h_{1}\right)\left(h_{2}\right)-f_{+}^{\prime}(x)\left(h_{2}\right)\right\}
$$

we obtain, using positive homogeneity,

$$
g_{+}^{\prime \prime}(x)(x ; h)=g_{+}^{\prime}(x)(h)=(x, h),
$$

and, directly from (6),

$$
g_{+}^{\prime \prime}(0)(x ; h)=(x, h)
$$

From the formula

$$
g(x+t h)-g(x)=1 / 2\{\|x+t \cdot h\|-\|x\|\}\{\|x+t \cdot h\|+\|x\|\}
$$

another useful representation is deduced:

$$
(x, h)=\|x\| \lim _{t \rightarrow 0+} \frac{1}{t}\{\|x+t h\|-\|x\|\} .
$$

From (9) the convexity of the generalized inner product in its second variable is most easily seen once more. This yields, by use of the abovementioned topology,

( $\beta$ ) The generalized inner product is a continuous function of its second variable.

Another immediate consequence of (9) is the generalized CauchySchwarz inequality

$$
|(x, h)| \leq\|x\| \cdot\|b\| \cdot
$$

2. Two lemmas. The following elementary remarks do not seem to be generally known, though they are useful:

( $y$ ) A normed space with dimension $\geq 2$ is an inner product space iff this bolds for every two-dimensional subspace.

(The lemma $(\gamma)$, with 3 instead of 2 , will be denoted by $(\bar{\gamma}) ;(\bar{\gamma})$ is trivial since in each of the axioms for an inner product there occur at most 3 vectors. Lemma $(\gamma)$ has been proved by the parallelogram equality of 
Jordan-von Neumann. There are geometrical proofs [5] characterizing the ellipsoids with center 0 as the only surfaces which meet each two-dimensional subspace in a central ellipse.) Another elementary consequence of the convexity of $g$ is:

$(\delta)$ In a two-dimensional normed space $g$ is differentiable at almost all unit vectors. (In other words, $(x, b)$ is a linear function of $b$ for $x \in$ $\mathbf{R} \cdot E$, where $E$ contains almost all unit vectors.)

\section{Proofs for Tapia's characterizations.}

Theorem 1. A normed space is an inner product space iff the generalized inner product $(x, b)$ is a linear function of $x$.

Proof. In view of $(\gamma)$ it will be sufficient to consider two-dimensional spaces. By $(\delta)$ we may choose a basis $\left\{e_{1}, e_{2}\right\}$ such that $\dot{g}$ is differentiable at $e_{1}, e_{2}$. Let $x=\xi_{1} e_{1}+\xi_{2} e_{2}, b \doteq \eta_{1} e_{1}+\eta_{2} e_{2}$; then the linearity assumption gives

$$
(x, h)=\sum \xi_{i}\left(e_{i}, h\right)=\sum \xi_{i} \eta_{k}\left(e_{i}, e_{k}\right),
$$

which is an inner product.

Theorem 2. A normed space is an inner product space iff $(x, b)=$ $(b, x)$ for all $x, h$.

Proof. If this symmetry holds, then by $(\delta)$ the function $(x, b)$ is linear in $x$ for almost all $b$, and by $(\beta)$ it is continuous in $b$, hence linear everywhere in $x$. Apply Theorem 1 .

Because of (8) the results may be reformulated:

Theorem 3. A normed space is an inner product space iff $g$ is twice Fréchet-differentiable at 0 . (Then all derivatives of $g$ exist at each point.)

4. Symmetry of orthogonality (transversality). The generalized inner product can be traced back to the beginning of our century in connection with the calculus of variations. Blaschke in his theorem [1] on the symmetry of transversality originally made smoothness assumptions.

Theorem 4. Let $V$ be a normed space of dimension $\geq 3$. Then $V$ is an inner product space iff $(x, b)=0$ implies $(b, x)=0$. (This does not hold for dimension 2; see [8].)

Proof. Consider a 2-dimensional subspace $U$ of $V$, and the indicatrix 
$I_{U}=\{x \in U \mid\|x\|=1\}$. Since this is convex, it has a unit tangent vector at each point: For $x \in I_{u}$ there exists $b \in I_{u}$ such that $(x, b)=0$. Writing $(b, x)=0$ for $(x, b)=0$, we get the statement that every unit vector is the tangent to $I_{U}$ at some point. But this means the curve $I_{U}$ is smooth, for if it had a corner (point with two linearly independent tangent vectors $\left.b, b^{\prime}\right)$ we could find a unit vector $b^{\prime \prime}$ nowhere tangent to $I_{U}$, take $b^{\prime \prime}=$ $\left(b-b^{\prime}\right) /\left\|b-b^{\prime}\right\|$.

The smoothness of the sections $I_{U}$ implies the smoothness of the whole indicatrix $I_{V}$. In particular, for each $x \in V$, the set $\{b \mid(x, b)=0\}$ is a subspace of $V$; hence ${ }^{1}$

$$
\{h \mid(h, x)=0\} \text { is a subspace of } V .
$$

We claim that condition (11) implies $V$ is an inner product space. By observation $(\bar{\gamma})$, that is $(\gamma)$ with 3 in place of 2 , it suffices to show this for $V$ 3-dimensional. In this case, (11) says that under parallel light of direction $x$ the line separating the dark part of the indicatrix surface from the bright part lies in the plane. ${ }^{2}$

By means of elementary geometry [1, pp. 157-158] this gives: Every two-dimensional section $I_{U}$ is affinely symmetric with respect to each of its diameters. Then $I_{U}$ is necessarily an ellipse, which is seen either as in [1, pp. 158-159] or without "euclidean" means using Loewner's ellipse of $I_{U}[3]$. The rest follows from $(\gamma)$.

In view of this proof Theorem 4 can be reformulated:

Theorem 5. Let $V$ be a normed vector space of dimension $\geq 3$. Then the following conditions are equivalent:

(a) $V$ is an inner product space.

(b) For all $x, b \in V,(x, b)=0 \Rightarrow(b, x)=0$.

(c) For all $x \in V,\{b \in V \mid(b, x)=0\}$ is a subspace of $V$.

(For 2-dimensional spaces, one has $(a) \Rightarrow(b) \Rightarrow(c)$, but not the reverse implications.)

\section{REFERENCES}

1. W. Blaschke, Kreis und Kugel, 2te Aufl., de Gruyter, Berlin, 1956, MR 17, 1123.

${ }^{1}$ I wish to thank the referee for simplifying my original proof up to this point. The reader will easily see that Theorem 4 also holds if the indicatrix is not centrally symmetric, which was included in the author's former proof. The referee also suggested the elegant formulation of Theorem 5 .

${ }^{2}$ The following arguments are not new; but former proofs of Theorem 4 always needed smoothness. See also [11, p. 187]. 
2. T. Bonnesen and W. Fenchel, Theorie der konvexen Körper, Springer, Berlin, 1934.

3. L. Danzer, D. Laugwitz und H. Lenz, Uber das Löwnersche Ellipsoid und sein Analogen unter den einem Eikörper einbeschiebenen Ellipsoiden, Arch. Math. 8 (1957), 214-219. MR 20 \#1283.

4. D. Laugwitz, Differentialgeometrie in Vektorräumen, unter besonderer Berücksichtigung der unendlichdimensionalen Räume, Vieweg, Braunschweig, 1965. MR $32 \# 406$.

5. - Konvexe Mittelpunktsbereiche und normierte Räume, Math. Z. 61 (1954), 235-244. MR 16, 613.

6. D. Laugwitz and E. R. Lorch, Riemann metrics associated with convex bodies and normed spaces, Amer. J. Math. 78 (1956), 889-894. MR 18, 495.

7. E. R. Lorch, A curvature study of convex bodies in Banach spaces, Ann. Mat. Pura Appl. (4) 34 (1953), 105-112. MR 14, 657.

8. J. Radon, Ûber eine besondere. Art ebener konvexer Kurven, Leipziger Ber. Math.-Phys. Kl. 68 (1916), 123-128.

9. H. Rund, The differential geometry of Finsler spaces, Die Grundlehren der math. Wissenschaften, Band 101, Springer-Verlag, Berlin, 1959. MR 21 \#4662.

10. R. A. Tapia, A characterization of inner product spaces, Bull. Amer. Math. Soc. 79 (1973), 530-531; MR 47 \#3964. Extended version, Proc. Amer. Math. Soc. 41 (1973), 569-574.

11. F. A. Valentine, Konvexe Mengen, Hochschultaschenbücher, Band 402/ 402a, Bibliographisches Institut, Mannheim, 1968. MR 37 \#2084.

FACHBEREICH MATHEMATIK, TECHNISCHE HOCHSCHULE, 61 DARMSTADT, FEDERAL REPUBLIC OF GERMANY 